\title{
THE NEGATIVE RESULT OF GRAVITATIONAL TESTS FOR MULTIDIMENSIONAL KALUZA-KLEIN MODELS
}

\author{
M. EINGORN, A. ZHUK \\ Astronomical Observatory and Department of Theoretical Physics, \\ Odessa National University \\ maxim. eingorn@ gmail. com, ai_zhuk2@ rambler. ru)
}

PACS 04.25.Nx, 04.50.Cd (2, Dvoryanskaya Str., Odessa 65082, Ukraine; e-mail:

(C) 2012

\begin{abstract}
In the Kaluza-Klein model with toroidal extra dimensions, we obtain the metric coefficients in the weak-field approximation for delta-shaped matter sources. These metric coefficients are used to calculate the formulas for the frequency shift, perihelion shift, and deflection of light. In the leading order of approximation, the formula for the frequency shift coincides with the well-known expression from general relativity. However, for the perihelion shift and the light deflection, we obtain formulas $D \pi r_{g} /\left[(D-2) a\left(1-e^{2}\right)\right]$ and $(D-1) r_{g} /[(D-2) \rho]$, respectively, where $D$ is a total number of spatial dimensions. These expressions demonstrate good agreement with experimental data only in the case of the ordinary three-dimensional $(D=3)$ space. This result does not depend on the size of the extra dimensions. We also obtain the exact 5D soliton solution with correct non-relativistic Newtonian limit. The energy momentum tensor for this solution has clear physical interpretation. However, the classical tests for this metric do not satisfy the experimental data. Therefore, the considered multidimensional Kaluza-Klein models face a severe problem.
\end{abstract}

\section{Introduction}

The idea of the multidimensionality of our Universe demanded by the theories of unification of the fundamental interactions is one of the most breathtaking ideas of theoretical physics. It takes its origin from the pioneering works by Th. Kaluza and O. Klein [1]. Now, the most self-consistent modern theories of unification such as superstrings, supergravity, and M-theory are constructed in spacetime with extra dimensions [2]. Different aspects of the idea of the multidimensionality are intensively used in numerous recent articles. Therefore, it is very important to suggest experiments which can reveal the extra dimensions. For example, one of the aims of the Large Hadronic Collider consists in detecting the
Kaluza-Klein particles which correspond to excitations of the internal spaces (see, e.g., [3]). On the other hand, if we can show that the existence of extra dimensions is contrary to observations, then these theories are prohibited. This important problem is extensively discussed in the scientific literature (see, e.g., [4-10]).

It is well known that classical gravitational tests such as the frequency shift, perihelion shift, and deflection of light are crucial tests of any gravitational theory. For example, there is the significant discrepancy for Mercury between the measurement value of the perihelion shift and its calculated value using Newton's formalism [11]. This indicates that non-relativistic Newton's theory of gravity is not complete. This problem was resolved with the help of general relativity which is in good agreement with observations. A similar situation happened with deflection of light [12]. Obviously, the multidimensional gravitational theories should also be in concordance with these experimental data. To check it, the corresponding estimates were carried out in a number of works. For example, in [8], it was investigated the well-known multidimensional black hole solution [13], and the authors obtained a negative result. However, this result was clear from the very beginning, because the solution [13] does not have non-relativistic Newtonian limit in the case of extra dimensions. Definitely, such solutions in the solar system lead to results, which are far from the experimental data. The 5 -D soliton metrics [14-16] were explored in [4-7]. In [5] and [6], the range of parameters, for which classical gravitational tests for these metrics satisfy the observational values, was found. The black string (see, e.g., [17]) is a particular limiting case of such solutions with a trivial metric coefficient for the extra dimension. How- 
ever, as we show in Section 4, this range of parameters is quite far from the values, which possess the correct non-relativistic Newtonian limit for a point mass gravitating source. Moreover, for the soliton metrics which belongs to this range of parameters, the energy momentum tensor has the form that is hard to imagine for ordinary astrophysical objects. For example, the black string has matter sources with non-zero tension (see, e.g., $[18,19])$. It is very difficult to explain how astrophysical objects, e.g. the Sun, can have such energy momentum tensor.

In the 5-D non-factorizable brane world model, classical gravitational tests were investigated in [20]. Here, the model contains one free parameter associated with the bulk Weyl tensor. For appropriate values of this parameter, the perihelion shift in this model does not contradict observations. Certainly, this result is of interest, and it is necessary to examine carefully this model to verify the naturalness of the conditions imposed.

In our paper, we consider the classical gravitational tests in Kaluza-Klein models with an arbitrary number of spatial dimensions $D \geq 3$. We suppose that, in the absence of gravitating masses, the metric is a flat one. Gravitating masses (moving or at rest) perturb this metric, and we consider these perturbations in the weak-field approximation. In this approximation, we obtain the asymptotic form of the metric coefficients. Then we admit that, first, the extra dimensions are compact and have the topology of a torus and, second, the gravitational potential far away from gravitating masses tends to the non-relativistic Newtonian limit. All our assumptions are very general and natural. In the case of a gravitating body at rest, the obtained metric coefficients are used to calculate the frequency shift, perihelion shift, and deflection of light. We demonstrate that, for the frequency shift type experiment, it is hardly possible to observe the difference between the usual four-dimensional general relativity and multidimensional Kaluza-Klein models. However, the situation is quite different for the perihelion shift and the deflection of light. In these two cases, we get formulas which generalize the corresponding ones in general relativity. We show that both of these formulas for the perihelion shift and the deflection of light depend on a total number of spatial dimensions, and they are in good agreement with observations only in the ordinary three-dimensional space. It is worth noting that this result does not depend explicitly on the size of extra dimensions. So, we cannot avoid the problem with the classical gravitational tests in the limit of an arbitrarily small size of extra dimen- sions. We mention that, in work [9], the authors arrived at the same conclusions within the different approach.

We also obtain the exact soliton solution with correct non-relativistic Newtonian limit. The metric coefficients in this limit exactly coincide with asymptotic expressions we found in the weak-field approximation for a delta-shaped gravitating source. Moreover, the tension is absent in this model, and the energy momentum tensor has a clear physical interpretation. However, the classical tests for this metric do not satisfy the experimental data. This is the predictable result, because the parameters of this solution do not belong to the range found in $[5,6]$ and discussed above.

Therefore, our results show that the considered multidimensional Kaluza-Klein models face a severe problem.

The paper is structured as follows. In Section 2, we get the asymptotic metric coefficients in the weak-field limit for the delta-shaped matter gravitating source. These metric coefficients are used to calculate the formulas of the frequency shift, perihelion shift, and deflection of light in Section 3. In Section 4, we obtain the exact soliton solution with proper boundary conditions. Here, we compare this solution with the black string solution. The main results are summarized and discussed in the concluding section 5 .

\section{Weak Gravitational Field Approximation}

To start with, we consider the general form of a multidimensional metric:

$$
\begin{aligned}
& d s^{2}=g_{i k} d x^{i} d x^{k}=g_{00}\left(d x^{0}\right)^{2}+ \\
& +2 g_{0 \alpha} d x^{0} d x^{\alpha}+g_{\alpha \beta} d x^{\alpha} d x^{\beta}
\end{aligned}
$$

where the Latin indices $i, k=0,1, \ldots, D$ and the Greek indices $\alpha, \beta=1, \ldots, D$, and $D$ is the total number of spatial dimensions. We make the natural assumption that, without matter sources, the spacetime is the Minkowski one: $g_{00}=\eta_{00}=1, g_{0 \alpha}=\eta_{0 \alpha}=0, g_{\alpha \beta}=\eta_{\alpha \beta}=-\delta_{\alpha \beta}$. At the same time, the extra dimensions may have the topology of a torus. In the presence of matter, the metric is not a Minkowskian one, and we will investigate it in the weak-field limit. This means that the gravitational field is weak, and the velocities of the test bodies are small compared to the speed of light $c$. In this case, the metric is only slightly perturbed from its flat spacetime value:

$g_{i k} \approx \eta_{i k}+h_{i k}$

where $h_{i k}$ are corrections of the order $1 / c^{2}$. In particular, $h_{00} \equiv 2 \varphi / c^{2}$. Later, we will demonstrate that $\varphi$ 
is the non-relativistic gravitational potential. The same conclusion with respect to $\varphi$ can be easily obtained from the comparison of the non-relativistic action of a test mass moving in a gravitational field with its relativistic action. To get the other correction terms up to the same order $1 / c^{2}$, we should consider the multidimensional Einstein equation

$R_{i k}=\frac{2 S_{D} \tilde{G}_{\mathcal{D}}}{c^{4}}\left(T_{i k}-\frac{1}{D-1} g_{i k} T\right)$,

where $S_{D}=2 \pi^{D / 2} / \Gamma(D / 2)$ is the total solid angle (surface area of the $(D-1)$-dimensional sphere of unit radius), and $\tilde{G}_{\mathcal{D}}$ is the gravitational constant in the $(\mathcal{D}=D+1)$-dimensional spacetime. We are going to investigate the weak-field approximation, where the gravitational field is generated by $N$ moving point masses. Therefore, the energy-momentum tensor is

$T^{i k}=\sum_{p=1}^{N} m_{p}\left[(-1)^{D} g\right]^{-1 / 2} \frac{d x^{i}}{d t} \frac{d x^{k}}{d t} \frac{c d t}{d s} \delta\left(\mathbf{r}-\mathbf{r}_{p}\right)$,

where $m_{p}$ is the rest mass, and $\mathbf{r}_{p}$ is the radius vector of the $p$-th particle, respectively. All radius vectors $\mathbf{r}$ and $\mathbf{r}_{p}$ are $D$-dimensional, e.g. $\mathbf{r}=\left(x^{1}, x^{2}, \ldots, x^{D}\right)$, where $x^{\alpha}$ are coordinates in metric (2.1). The rest mass density is

$\rho \equiv \sum_{p=1}^{N} m_{p} \delta\left(\mathbf{r}-\mathbf{r}_{p}\right)$

\section{1. $1 / c^{2}$ correction terms}

Obviously, to hold the terms up to the order $1 / c^{2}$ on the right-hand side of (2.3), the components of the energymomentum tensor (2.4) are approximated as

$T_{00} \approx \rho c^{2}, \quad T_{0 \alpha} \approx 0, \quad T_{\alpha \beta} \approx 0 \Rightarrow T=T_{i}^{i} \approx \rho c^{2}$.

Taking into account that $h_{i k}$ are of the order of $1 / c^{2}$, the covariant components of the Riemann and Ricci tensors

$$
\begin{aligned}
& R_{i k l m}=\frac{1}{2}\left(\frac{\partial^{2} g_{i m}}{\partial x^{k} \partial x^{l}}+\frac{\partial^{2} g_{k l}}{\partial x^{i} \partial x^{m}}-\frac{\partial^{2} g_{i l}}{\partial x^{k} \partial x^{m}}-\right. \\
& \left.-\frac{\partial^{2} g_{k m}}{\partial x^{i} \partial x^{l}}\right)+g_{n p}\left(\Gamma_{k l}^{n} \Gamma_{i m}^{p}-\Gamma_{k m}^{n} \Gamma_{i l}^{p}\right) \\
& R_{k m}=g^{i l} R_{i k l m}
\end{aligned}
$$

up to the same order read

$$
\begin{aligned}
& R_{i k l m} \approx \frac{1}{2}\left(\frac{\partial^{2} h_{i m}}{\partial x^{k} \partial x^{l}}+\frac{\partial^{2} h_{k l}}{\partial x^{i} \partial x^{m}}-\right. \\
& \left.-\frac{\partial^{2} h_{i l}}{\partial x^{k} \partial x^{m}}-\frac{\partial^{2} h_{k m}}{\partial x^{i} \partial x^{l}}\right),
\end{aligned}
$$

$$
\begin{aligned}
& R_{k m} \approx \frac{1}{2} \eta^{i l}\left(\frac{\partial^{2} h_{i m}}{\partial x^{k} \partial x^{l}}+\frac{\partial^{2} h_{k l}}{\partial x^{i} \partial x^{m}}-\frac{\partial^{2} h_{i l}}{\partial x^{k} \partial x^{m}}-\right. \\
& \left.-\frac{\partial^{2} h_{k m}}{\partial x^{i} \partial x^{l}}\right)=\frac{1}{2}\left(\frac{\partial^{2} h_{m}^{l}}{\partial x^{k} \partial x^{l}}+\frac{\partial^{2} h_{k}^{l}}{\partial x^{m} \partial x^{l}}-\right. \\
& \left.-\frac{\partial^{2} h_{l}^{l}}{\partial x^{k} \partial x^{m}}-\eta^{i l} \frac{\partial^{2} h_{k m}}{\partial x^{i} \partial x^{l}}\right),
\end{aligned}
$$

where $h_{k}^{i} \equiv \eta^{i m} h_{m k}$. With the help of the gauge conditions

$$
\frac{\partial}{\partial x^{k}}\left(h_{i}^{k}-\frac{1}{2} h_{l}^{l} \delta_{i}^{k}\right)=0
$$

formula (2.9) can be written in the form

$R_{k m} \approx-\frac{1}{2} \eta^{i l} \frac{\partial^{2} h_{k m}}{\partial x^{i} \partial x^{l}}$

Taking into account that the derivatives with respect to $x^{0}=c t$ are much smaller than the derivatives with respect to $x^{\alpha}$, relation $(2.11)$ yields

$R_{00} \approx-\frac{1}{2} \eta^{\alpha \beta} \frac{\partial^{2} h_{00}}{\partial x^{\alpha} \partial x^{\beta}}=\frac{1}{2} \delta^{\alpha \beta} \frac{\partial^{2} h_{00}}{\partial x^{\alpha} \partial x^{\beta}}=\frac{1}{2} \triangle h_{00}$,

$R_{0 \alpha} \approx \frac{1}{2} \triangle h_{0 \alpha}, \quad R_{\alpha \beta} \approx \frac{1}{2} \triangle h_{\alpha \beta}$,

where $\triangle=\delta^{\alpha \beta} \partial^{2} / \partial x^{\alpha} \partial x^{\beta}$ is the $D$-dimensional Laplace operator. It is worth noting that condition (2.10) holds up to the order $1 / c^{2}$,

$$
\begin{aligned}
& \frac{\partial}{\partial x^{\beta}}\left(h_{\alpha}^{\beta}-\frac{1}{2} h_{l}^{l} \delta_{\alpha}^{\beta}\right)=0+O\left(1 / c^{3}\right), \\
& \frac{\partial h_{0}^{\beta}}{\partial x^{\beta}}=0+O\left(1 / c^{3}\right) .
\end{aligned}
$$

Therefore, keeping the terms up to the order $1 / c^{2}$ on the left- and right-hand sides of (2.3), we obtain the equations

$$
\begin{aligned}
\triangle h_{00} & =\frac{2 S_{D} G_{\mathcal{D}}}{c^{2}} \rho, \quad \Delta h_{0 \alpha}=0, \\
\triangle h_{\alpha \beta} & =\frac{1}{D-2} \cdot \frac{2 S_{D} G_{\mathcal{D}}}{c^{2}} \rho \delta_{\alpha \beta},
\end{aligned}
$$

where $G_{\mathcal{D}}=[2(D-2) /(D-1)] \tilde{G}_{\mathcal{D}}$. The substitution of $h_{00}=2 \varphi / c^{2}$ into the above equation for $h_{00}$ demonstrates that $\varphi$ satisfies the $D$-dimensional Poisson equation:

$\triangle \varphi=S_{D} G_{\mathcal{D}} \rho$ 
Therefore, $\varphi$ is a non-relativistic gravitational potential. From (2.15), we obtain

$h_{0 \alpha}=0, \quad h_{\alpha \beta}=\frac{1}{D-2} h_{00} \delta_{\alpha \beta}=\frac{1}{D-2} \frac{2 \varphi}{c^{2}} \delta_{\alpha \beta}$.

It can be easily seen that, in this approximation, the spatial coordinates of metric (2.1) are the isotropic ones, i.e. the spatial part of the metric is conformally related to the Euclidean one. We note also that the relation $h_{\alpha \beta} / h_{00}=[1 /(D-2)] \delta_{\alpha \beta}$ can be also obtained from the corresponding equations in works $[13,21]$.

\section{2. $1 / c^{3}$ and $1 / c^{4}$ correction terms}

Now, we want to keep the terms up to the order $1 / c^{2}$ in metric (2.1). Because the coordinate $x^{0}=c t$ contains $c$, this means that, in $g_{00}$ and $g_{0 \alpha}$, we should keep correction terms up to the order $1 / c^{4}$ and $1 / c^{3}$, respectively, and to leave $g_{\alpha \beta}$ without changes in the form $g_{\alpha \beta} \approx \eta_{\alpha \beta}+h_{\alpha \beta}$ with $h_{\alpha \beta}$ from (2.17).

First, we investigate the energy-momentum tensor (2.4), which we split into three expressions:

$T^{00}=\sum_{p=1}^{N} m_{p} c^{2}\left[(-1)^{D} g\right]^{-1 / 2} \frac{c d t}{d s} \delta\left(\mathbf{r}-\mathbf{r}_{p}\right)$,

$T^{0 \alpha}=\sum_{p=1}^{N} m_{p} c\left[(-1)^{D} g\right]^{-1 / 2} v_{p}^{\alpha} \frac{c d t}{d s} \delta\left(\mathbf{r}-\mathbf{r}_{p}\right)$,

$T^{\alpha \beta}=\sum_{p=1}^{N} m_{p}\left[(-1)^{D} g\right]^{-1 / 2} v_{p}^{\alpha} v_{p}^{\beta} \frac{c d t}{d s} \delta\left(\mathbf{r}-\mathbf{r}_{p}\right)$

where $v_{p}^{\alpha}=d x_{p}^{\alpha} / d t$. From (2.20) up to order 1 (in units of $c$ ), we obtain the covariant components

$T_{\alpha \beta} \approx \sum_{p=1}^{N} m_{p} v_{p \alpha} v_{p \beta} \delta\left(\mathbf{r}-\mathbf{r}_{p}\right)$

Thus, with regard for the prefactor $1 / c^{4}$ on the righthand side of (2.3), these components can contribute terms of the order of $1 / c^{4}$ to $g_{\alpha \beta}$, which are not of interest for us. For $T_{0 \alpha}$, relation (2.19) yields

$T_{0 \alpha} \approx-\sum_{p=1}^{N} m_{p} c v_{p \alpha} \delta\left(\mathbf{r}-\mathbf{r}_{p}\right)$
Hence, these components can give terms of the order of $1 / c^{3}$ into $g_{0 \alpha}$. Finally, we obtain the following expression from (2.18) for $T_{00}$ :

$$
\begin{aligned}
& T_{00}=g_{0 i} g_{0 k} T^{i k} \approx \sum_{p=1}^{N} m_{p} c^{2} \delta\left(\mathbf{r}-\mathbf{r}_{p}\right)+ \\
& +\sum_{p=1}^{N} m_{p}\left(\frac{3 D-4}{D-2} \varphi_{p}+\frac{1}{2} v_{p}^{2}\right) \delta\left(\mathbf{r}-\mathbf{r}_{p}\right)
\end{aligned}
$$

where $\varphi_{p}$ is the potential of the gravitational field at a point with radius vector $\mathbf{r}_{p}$. At the moment, we do not care about the fact that $\varphi_{p}$ contains the infinite contribution of the $p$-th particle. Thus, up to order 1 , we get

$$
\begin{aligned}
& T=g^{i k} T_{i k} \approx \sum_{p=1}^{N} m_{p} c^{2} \delta\left(\mathbf{r}-\mathbf{r}_{p}\right)+ \\
& +\sum_{p=1}^{N} m_{p}\left(\frac{D}{D-2} \varphi_{p}-\frac{1}{2} v_{p}^{2}\right) \delta\left(\mathbf{r}-\mathbf{r}_{p}\right)
\end{aligned}
$$

With the help of (2.23) and (2.24), we obtain, up to the order $1 / c^{4}$,

$$
\begin{aligned}
& \frac{2 S_{D} \tilde{G}_{\mathcal{D}}}{c^{4}}\left(T_{00}-\frac{1}{D-1} g_{00} T\right) \approx \\
& \approx \frac{S_{D} G_{\mathcal{D}}}{c^{2}} \sum_{p=1}^{N} m_{p} \delta\left(\mathbf{r}-\mathbf{r}_{p}\right)+ \\
& +\frac{S_{D} G_{\mathcal{D}}}{c^{4}} \sum_{p=1}^{N} m_{p}\left(\frac{3 D-4}{D-2} \varphi_{p}+\right. \\
& \left.+\frac{D}{2(D-2)} v_{p}^{2}\right) \delta\left(\mathbf{r}-\mathbf{r}_{p}\right) .
\end{aligned}
$$

Similarly, from (2.22) and (2.24), we get (up to the order $\left.1 / c^{3}\right)$

$$
\begin{aligned}
& \frac{2 S_{D} \tilde{G}_{\mathcal{D}}}{c^{4}}\left(T_{0 \alpha}-\frac{1}{D-1} g_{0 \alpha} T\right) \approx \\
& \approx-\frac{D-1}{D-2} \frac{S_{D} G_{\mathcal{D}}}{c^{3}} \sum_{p=1}^{N} m_{p} v_{p \alpha} \delta\left(\mathbf{r}-\mathbf{r}_{p}\right) .
\end{aligned}
$$

Now, we work out the left-hand side of (2.3) up to the appropriate orders of $1 / c$. As we wrote above, we are looking for corrections of the order of $1 / c^{4}$ and $1 / c^{3}$ to the metric components $g_{00}$ and $g_{0 \alpha}$, respectively. To this end, it is convenient to present $g_{i k}$ as follows:

$g_{i k} \approx \eta_{i k}+h_{i k}+f_{i k}$ 
where $f_{00}$ and $f_{0 \alpha}$ are of the order of $1 / c^{4}$ and $1 / c^{3}$, respectively. Then the 00-component of the Ricci tensor up to the order $1 / c^{4}$ reads

$R_{00} \approx \frac{1}{c^{2}} \triangle \varphi+\frac{1}{2} \triangle f_{00}+\frac{1}{D-2} \cdot \frac{2}{c^{4}} \varphi \Delta \varphi-\frac{2}{c^{4}}(\nabla \varphi)^{2}$,

where we take into account that $h_{0}^{\alpha}=h_{\alpha}^{0} \equiv 0$ and use the gauge condition

$\frac{\partial f_{0}^{\beta}}{\partial x^{\beta}}=\frac{1}{2} \frac{\partial h_{\beta}^{\beta}}{\partial x^{0}}$

and condition (2.14). The $0 \alpha$-component of the Ricci tensor up to the order $1 / c^{3}$ reads

$R_{0 \alpha} \approx \frac{1}{2} \triangle f_{0 \alpha}+\frac{1}{2 c^{3}} \frac{\partial^{2} \varphi}{\partial t \partial x^{\alpha}}$.

Now, we come back to the Einstein equation (2.3). Substituting (2.25) and (2.28) into (2.3) and taking (2.5) and (2.16) into account, we get the following equation for $f_{00}$ :

$\triangle f_{00}+\frac{1}{D-2} \frac{4}{c^{4}} \varphi \triangle \varphi-\frac{4}{c^{4}}(\nabla \varphi)^{2}=$

$=\frac{2 S_{D} G_{\mathcal{D}}}{c^{4}} \sum_{p=1}^{N} m_{p}\left(\frac{3 D-4}{D-2} \varphi_{p}+\frac{D}{2(D-2)} v_{p}^{2}\right) \times$

$\times \delta\left(\mathbf{r}-\mathbf{r}_{p}\right)$.

With the help of the auxiliary equation

$4(\nabla \varphi)^{2}=2 \triangle\left(\varphi^{2}\right)-4 \varphi \triangle \varphi$

and Eqs. (2.5) and (2.16), Eq. (2.31) takes the form

$\triangle\left(f_{00}-\frac{2}{c^{4}} \varphi^{2}\right)=\frac{2 S_{D} G_{\mathcal{D}}}{c^{4}} \times$

$\times \sum_{p=1}^{N} m_{p}\left(\varphi_{p}^{\prime}+\frac{D}{2(D-2)} v_{p}^{2}\right) \delta\left(\mathbf{r}-\mathbf{r}_{p}\right)$.

Here, $\varphi_{p}^{\prime}$ is the potential of the gravitational field at a point with radius vector $\mathbf{r}_{p}$ produced by all particles, except for the $p$-th one. The subtraction of the infinite contribution of the gravitational field of the $p$-th particle corresponds to a renormalization of its mass (see [22]). The solution of $(2.33)$ is

$$
\begin{aligned}
& f_{00}=\frac{2}{c^{4}} \varphi^{2}(\mathbf{r})+\frac{2}{c^{4}} \sum_{p=1}^{N} \varphi_{p}^{\prime} \varphi^{\prime}\left(\mathbf{r}-\mathbf{r}_{p}\right)+ \\
& +\frac{D}{D-2} \cdot \frac{1}{c^{4}} \sum_{p=1}^{N} v_{p}^{2} \varphi^{\prime}\left(\mathbf{r}-\mathbf{r}_{p}\right)
\end{aligned}
$$

where $\varphi^{\prime}\left(\mathbf{r}-\mathbf{r}_{p}\right)$ is the potential of the gravitational field of the $p$-th particle which satisfies the Poisson equation

$\triangle \varphi^{\prime}=\delta^{\alpha \beta} \frac{\partial^{2} \varphi^{\prime}}{\partial x^{\alpha} \partial x^{\beta}}=S_{D} G_{\mathcal{D}} m_{p} \delta\left(\mathbf{r}-\mathbf{r}_{p}\right)$

It can be easily verified with the help of (2.5) and (2.16) that $\varphi^{\prime}\left(\mathbf{r}-\mathbf{r}_{p}\right)$ satisfies the condition

$\varphi(\mathbf{r})=\sum_{p=1}^{N} \varphi^{\prime}\left(\mathbf{r}-\mathbf{r}_{p}\right)$

Therefore, substituting $h_{00}=2 \varphi / c^{2}$ and $f_{00}$ into (2.27), we obtain $g_{00}$ up to the order $1 / c^{4}$ :

$g_{00} \approx 1+\frac{2}{c^{2}} \varphi(\mathbf{r})+\frac{2}{c^{4}} \varphi^{2}(\mathbf{r})+\frac{2}{c^{4}} \sum_{p=1}^{N} \varphi_{p}^{\prime} \varphi^{\prime}\left(\mathbf{r}-\mathbf{r}_{p}\right)+$

$+\frac{D}{D-2} \frac{1}{c^{4}} \sum_{p=1}^{N} v_{p}^{2} \varphi^{\prime}\left(\mathbf{r}-\mathbf{r}_{p}\right)$.

We should mention that the radius vectors $\mathbf{r}_{p}$ of the moving gravitating masses depend on the time. In this case, the potential $\varphi(\mathbf{r})$ in (2.36) also depends on the time.

The equation for $f_{0 \alpha}$ can be obtained by the substitution of (2.26) and (2.30) into the Einstein equation $(2.3)$ :

$\triangle f_{0 \alpha}=-\frac{2(D-1)}{D-2} \frac{S_{D} G_{\mathcal{D}}}{c^{3}} \sum_{p=1}^{N} m_{p} v_{p \alpha} \delta\left(\mathbf{r}-\mathbf{r}_{p}\right)-$

$-\frac{1}{c^{3}} \frac{\partial^{2} \varphi}{\partial t \partial x^{\alpha}}$

whose solution is

$f_{0 \alpha}=-\frac{2(D-1)}{D-2} \frac{1}{c^{3}} \sum_{p=1}^{N} v_{p \alpha} \varphi^{\prime}\left(\mathbf{r}-\mathbf{r}_{p}\right)-\frac{1}{c^{3}} \frac{\partial^{2} f}{\partial t \partial x^{\alpha}}$

where the function $f$ satisfies the equation

$\triangle f=\delta^{\alpha \beta} \frac{\partial^{2} f}{\partial x^{\alpha} \partial x^{\beta}}=\varphi(\mathbf{r})$.

Therefore, substituting $h_{0 \alpha}=0$ and $f_{0 \alpha}$ into (2.27), we get $g_{0 \alpha}$ up to the order $1 / c^{3}$ :

$g_{0 \alpha} \approx-\frac{2(D-1)}{D-2} \frac{1}{c^{3}} \sum_{p=1}^{N} v_{p \alpha} \varphi^{\prime}\left(\mathbf{r}-\mathbf{r}_{p}\right)-\frac{1}{c^{3}} \frac{\partial^{2} f}{\partial t \partial x^{\alpha}}$ 
We note that, in the three-dimensional case $D=3$, relations (2.37) and (2.41) exactly coincide with (106.13) and (106.14) in [22] if we take into account that $\varphi^{\prime}(\mathbf{r}-$ $\left.\mathbf{r}_{p}\right)=-G_{N} m_{p} /\left|\mathbf{r}-\mathbf{r}_{p}\right|$.

We now consider the case of one gravitating particle of mass $m_{1} \equiv m$, which is at rest in our $3-\mathrm{D}$ space but, for generality, moves with constant speed in extra dimensions. That is, $p=1 \Rightarrow \varphi_{1}^{\prime}=0$ and $v^{\alpha}=d x^{\alpha} / d t=\left(0,0,0, v_{4}, v_{5}, \ldots, v_{D}\right)$, where $v_{4}, v_{5}, \ldots, v_{D}$ are constants. In this case, (2.37) and (2.41) are reduced, respectively, to

$g_{00} \approx 1+\frac{2}{c^{2}} \varphi(\mathbf{r})+\frac{2}{c^{4}} \varphi^{2}(\mathbf{r})+\frac{D v^{2}}{(D-2) c^{4}} \varphi(\mathbf{r})$

and

$g_{0 \alpha} \approx-\frac{2(D-1) v_{\alpha}}{(D-2) c^{3}} \varphi(\mathbf{r})-\frac{1}{c^{3}} \frac{\partial^{2} f}{\partial t \partial x^{\alpha}}$,

where $\varphi(\mathbf{r})$ satisfies the Poisson equation

$\triangle \varphi=\delta^{\alpha \beta} \frac{\partial^{2} \varphi}{\partial x^{\alpha} \partial x^{\beta}}=S_{D} G_{\mathcal{D}} m \delta(\mathbf{r})$,

and $v^{2}=-g_{\alpha \beta} v^{\alpha} v^{\beta}=v_{4}^{2}+v_{5}^{2}+\ldots+v_{D}^{2}+O\left(1 / c^{2}\right)$ (at the same accuracy, $\left.v_{\beta}=-v^{\beta}\right)$. Obviously, the transition to the case where the gravitating mass is at rest both in our three-dimensional space and in the extra dimensions corresponds to the limit $v_{\alpha}=0 \Rightarrow v^{2}=0$. In this case, the potential $\varphi(\mathbf{r})$ and the function $f$ do not depend on the time $t$. We recall that the covariant components $g_{\alpha \beta}$ read (see (2.17))

$g_{\alpha \beta} \approx-\left(1-\frac{1}{D-2} \cdot \frac{2}{c^{2}} \varphi(\mathbf{r})\right) \delta_{\alpha \beta}$.

To get all above results, we did not use any specific form of topology. The only things we used were assumptions of the flatness of the metric in the absence of gravitating masses and the weakness of the gravitational field and velocities of gravitating masses, which perturb the flat metric. Now, to solve (2.44), we should specify the topology of a space and the boundary conditions. We suppose that the $(D=3+d)$-dimensional space has the factorizable geometry of a product manifold $M_{D}=\mathbb{R}^{3} \times T^{d}$. The quantity $\mathbb{R}^{3}$ describes the three-dimensional flat external (our) space, and $T^{d}$ is a torus, which corresponds to a $d$-dimensional internal space with volume $V_{d}$. For this topology and with the boundary condition that the potential must go to the Newtonian expression at infinitely large distances from the gravitating body, we can find the exact solution of the Poisson equation (2.44) [23,24]. The boundary condition requires that the multidimensional $G_{\mathcal{D}}$ and Newtonian $G_{N}$ gravitational constants be connected by the condition $S_{D} G_{\mathcal{D}} / V_{d}=4 \pi G_{N}$. Assuming that we consider the gravitational field of a gravitating mass $m$ at distances much greater than the periods of the torus, we can restrict ourselves to the zero Kaluza-Klein mode. For example, this approximation is very well satisfied for the planets of the solar system, because the inversesquare law experiments show that the extra dimensions in Kaluza-Klein models should not exceed submillimeter scales [25] (see, however, [23, 24] for models with smeared extra dimensions, where Newton's law preserves its shape for arbitrary distances). Then, the gravitational potential reads

$\varphi(\mathbf{r}) \approx-\frac{G_{N} m}{r_{3}}=-\frac{r_{g} c^{2}}{2 r_{3}}$

where $r_{3}$ is the length of a radius vector in the three-dimensional space, and we introduce the threedimensional Schwarzschild radius $r_{g}=2 G_{N} m / c^{2}$. As was mentioned above, the gravitating mass $m$ is at rest in our three-dimensional space, but it can move in the extra dimensions. In this case, the extra dimensional components of $D$-dimensional radius vector of a gravitating particle depend on the time. The exact formulas for the non-relativistic gravitational potential (see $[23,24])$ show that this dependence "nests" only in nonzero Kaluza-Klein modes, which are exponentially suppressed in the considered approximation. Therefore, in this approximation, the potential $\varphi(\mathbf{r})$ in $(2.46)$ does not depend on the time.

It is worth noting that all the previous analysis works also in the case where the gravitating masses are uniformly smeared over some or all extra dimensions. Let us take, for simplicity, one $(p=1)$ gravitating mass $m_{1} \equiv m$, which is smeared over all extra dimensions. Obviously, this mass can move only in our usual three dimensions: $v_{1}^{\alpha}=d x_{1}^{\alpha} / d t=\left(v_{1}^{1}, v_{1}^{2}, v_{1}^{3}, 0, \ldots, 0\right)$, and its rest mass density (2.5) now reads

$\rho=\left(m / \prod_{\alpha=1}^{d} a_{\alpha}\right) \delta\left(\mathbf{r}_{3}-\mathbf{r}_{(1) 3}\right)$

where $a_{\alpha}$ are the periods of the torus. Then, the solution of the Poisson equation (2.16) exactly coincides with the Newton potential if the multidimensional $G_{\mathcal{D}}$ and Newtonian $G_{N}$ gravitational constants are connected as $S_{D} G_{\mathcal{D}} / \prod_{\alpha=1}^{d} a_{\alpha}=4 \pi G_{N}[23,24]$. Therefore, in this case, the approximate formula (2.46) becomes the exact 
equality:

$\varphi(\mathbf{r})=\varphi\left(\mathbf{r}_{3}\right)=-\frac{G_{N} m}{r_{3}}=-\frac{r_{g} c^{2}}{2 r_{3}}$.

In approximation (2.46) (or with (2.48) for "smeared" extra dimensions), the covariant components (2.42), (2.43), and (2.45) take the form

$$
\begin{aligned}
& g_{00} \approx 1-\frac{r_{g}}{r_{3}}+\frac{r_{g}^{2}}{2 r_{3}^{2}}-\frac{D v^{2}}{2(D-2) c^{2}} \frac{r_{g}}{r_{3}}, \\
& g_{0 \alpha} \approx \frac{(D-1) v_{\alpha}}{(D-2) c} \frac{r_{g}}{r_{3}}, \\
& g_{\alpha \beta} \approx-\left(1+\frac{1}{D-2} \frac{r_{g}}{r_{3}}\right) \delta_{\alpha \beta} .
\end{aligned}
$$

For the contravariant components, we obtain

$$
\begin{aligned}
& g^{00} \approx 1+\frac{r_{g}}{r_{3}}+\frac{r_{g}^{2}}{2 r_{3}^{2}}+\frac{D v^{2}}{2(D-2) c^{2}} \frac{r_{g}}{r_{3}}, \\
& g^{0 \alpha} \approx-\frac{(D-1) v^{\alpha}}{(D-2) c} \frac{r_{g}}{r_{3}} \\
& g^{\alpha \beta} \approx-\left(1-\frac{1}{D-2} \frac{r_{g}}{r_{3}}\right) \delta_{\alpha \beta} .
\end{aligned}
$$

It is not difficult to verify that these components satisfy the condition

$g_{i k} g^{k j}=\left(\begin{array}{cc}1+O\left(1 / c^{6}\right) & 0+O\left(1 / c^{5}\right) \\ 0+O\left(1 / c^{5}\right) & \delta_{\alpha \beta}+O\left(1 / c^{4}\right)\end{array}\right)$.

The metric components (2.49) demonstrate that, in this approximation, the spatial section $t=$ const is conformal to the Euclidean metric. Hence, the spatial coordinates are isotropic ones. It is convenient to use the three-dimensional spherical coordinates $r_{3}, \theta, \psi$ instead of the Cartesian coordinates $x^{1} \equiv x, x^{2} \equiv y, x^{3} \equiv z$. In these coordinates, metric (2.1) reads

$$
\begin{aligned}
& d s^{2} \approx\left(1-\frac{r_{g}}{r_{3}}+\frac{r_{g}^{2}}{2 r_{3}^{2}}-\frac{D v^{2}}{2(D-2) c^{2}} \frac{r_{g}}{r_{3}}\right) c^{2} d t^{2}+ \\
& +\frac{2(D-1)}{(D-2) c} \frac{r_{g}}{r_{3}} c d t \sum_{\alpha=4}^{D} v_{\alpha} d x^{\alpha}- \\
& -\left(1+\frac{1}{D-2} \frac{r_{g}}{r_{3}}\right)\left(d r_{3}^{2}+r_{3}^{2} d \theta^{2}+r_{3}^{2} \sin ^{2} \theta d \psi^{2}\right)- \\
& -\left(1+\frac{1}{D-2} \frac{r_{g}}{r_{3}}\right)\left(\left(d x^{4}\right)^{2}+\right. \\
& \left.+\left(d x^{5}\right)^{2}+\ldots+\left(d x^{D}\right)^{2}\right) .
\end{aligned}
$$

As was mentioned above, this metric corresponds to a gravitating mass in the rest in our three-dimensional space. If the mass is smeared over extra dimensions, the appropriate velocity components vanish.

\section{Three Classical Tests}

We now check the above-obtained multidimensional metric (2.52) from the viewpoint of its consistency with the famous classical tests: the frequency shift, perihelion shift, and deflection of light. It is well known that the four-dimensional general relativity is in good agreement with these experiments. But is it true for the considered Kaluza-Klein models?

\subsection{Frequency shift}

To investigate the gravitational redshift formula (2.52) in the spacetime, we can use the famous expression for relation between the frequency $\omega_{1}$ of a light signal, which is emitted at point 1 with the metric component $\left.g_{00}\right|_{1}$ and the frequency $\omega_{2}$ and is received at point 2 with the metric component $\left.g_{00}\right|_{2}$ :

$\omega_{1}\left[\left(g_{00}\right)^{1 / 2}\right]_{1}=\omega_{2}\left[\left(g_{00}\right)^{1 / 2}\right]_{2}$.

Therefore, up to the order $1 / c^{2}$, we get

$\omega_{2} \approx \omega_{1}\left(1+\frac{\varphi_{1}-\varphi_{2}}{c^{2}}\right)$

where the non-relativistic potential $\varphi$ is given by (2.46). In the considered approximation, this formula exactly coincides with that of general relativity. Therefore, for this type of experiments, it is hardly possible to observe the difference between the usual four-dimensional general relativity and the multidimensional Kaluza-Klein models.

\subsection{Perihelion shift}

Let us consider the motion of a test body of mass $m^{\prime}$ in the gravitational field described by metric (2.52). The Hamilton-Jacobi equation

$g^{i k} \frac{\partial S}{\partial x^{i}} \frac{\partial S}{\partial x^{k}}-m^{\prime 2} c^{2}=0$

for this test body moving in the orbital plane $\theta=\pi / 2$ reads

$$
\begin{aligned}
& \frac{1}{c^{2}}\left(1+\frac{r_{g}}{r_{3}}+\frac{r_{g}^{2}}{2 r_{3}^{2}}+\frac{D v^{2}}{2(D-2) c^{2}} \frac{r_{g}}{r_{3}}\right)\left(\frac{\partial S}{\partial t}\right)^{2}- \\
& -\frac{2(D-1) v^{\alpha}}{(D-2) c^{2}} \frac{r_{g}}{r_{3}} \frac{\partial S}{\partial t} \frac{\partial S}{\partial x^{\alpha}}-\left(1-\frac{1}{D-2} \frac{r_{g}}{r_{3}}\right)\left(\frac{\partial S}{\partial r_{3}}\right)^{2}- \\
& -\frac{1}{r_{3}^{2}}\left(1-\frac{1}{D-2} \frac{r_{g}}{r_{3}}\right)\left(\frac{\partial S}{\partial \psi}\right)^{2}-\left(1-\frac{1}{D-2} \frac{r_{g}}{r_{3}}\right) \times \\
& \times\left[\left(\frac{\partial S}{\partial x^{4}}\right)^{2}+\ldots+\left(\frac{\partial S}{\partial x^{D}}\right)^{2}\right]-m^{\prime 2} c^{2} \approx 0
\end{aligned}
$$


We investigate this equation by the separation of variables considering the action in the form

$$
S=-E^{\prime} t+M \psi+S_{r_{3}}\left(r_{3}\right)+S_{4}\left(x^{4}\right)+\ldots+S_{D}\left(x^{D}\right) .
$$

Here, $E^{\prime} \approx m^{\prime} c^{2}+E$ is the energy of the test body, which includes the rest energy $m^{\prime} c^{2}$ and the non-relativistic en$\operatorname{ergy} E$, and $M$ is the angular momentum. Substituting this expression for the action $S$ in formula (3.4), we obtain an expression for $\left(d S_{r_{3}} / d r_{3}\right)^{2}$ holding the terms up to the order $1 / c^{2}$ :

$$
\begin{aligned}
& \left(\frac{d S_{r_{3}}}{d r_{3}}\right)^{2} \approx\left(2 m^{\prime} E-\left(p_{4}^{2}+\ldots+p_{D}^{2}\right)+\frac{E^{2}}{c^{2}}\right)- \\
& -\frac{1}{r_{3}^{2}}\left(M^{2}-\frac{D m^{\prime 2} c^{2} r_{g}^{2}}{2(D-2)}\right)+ \\
& +\frac{1}{r_{3}}\left(m^{\prime 2} c^{2} r_{g}+\frac{2(D-1)}{D-2} m^{\prime} E r_{g}+\right. \\
& \left.+\frac{D}{2(D-2)} m^{\prime 2} r_{g} v^{2}+\frac{2(D-1)}{(D-2)} m^{\prime} r_{g} \sum_{\alpha=4}^{D} v^{\alpha} p_{\alpha}\right),
\end{aligned}
$$

where $p_{\alpha}=\partial S / \partial x^{\alpha}=d S_{\alpha} / d x^{\alpha}(\alpha=4, \ldots, D)$ are the components of the momentum of the test body in the extra dimensions. If the gravitating and test masses are localized on the same brane, then these components are equal to zero. Integrating the square root of this expression with respect to $r_{3}$, we get $S_{r_{3}}$ in the form

$$
\begin{aligned}
& S_{r_{3}} \approx \int\left[\left(2 m^{\prime} E-\left(p_{4}^{2}+\ldots+p_{D}^{2}\right)+\frac{E^{2}}{c^{2}}\right)+\right. \\
& +\frac{1}{r_{3}}\left(m^{\prime 2} c^{2} r_{g}+\frac{2(D-1)}{D-2} m^{\prime} E r_{g}+\right. \\
& \left.+\frac{D}{2(D-2)} m^{\prime 2} r_{g} v^{2}+\frac{2(D-1)}{(D-2)} m^{\prime} r_{g} \sum_{\alpha=4}^{D} v^{\alpha} p_{\alpha}\right)- \\
& \left.-\frac{1}{r_{3}^{2}}\left(M^{2}-\frac{D m^{\prime 2} c^{2} r_{g}^{2}}{2(D-2)}\right)\right]^{1 / 2} d r_{3} .
\end{aligned}
$$

It is well known (see, e.g., $§ 47$ in [26]) that, for any integral of motion $I$ of a system with action $S$, the following equation should hold:

$$
\frac{\partial S}{\partial I}=\text { const } .
$$

Because the angular momentum $M$ is the integral of motion, the trajectory of a test body is defined by the equation

$$
\frac{\partial S}{\partial M}=\psi+\frac{\partial S_{r_{3}}}{\partial M}=\text { const }
$$

where we use (3.5)

Let now the Sun be the gravitating mass, and let the planets of the solar system be the test bodies. Then the change of the angle during one revolution of a planet on its orbit is

$\Delta \psi=-\frac{\partial}{\partial M} \Delta S_{r_{3}}$,

where $\Delta S_{r_{3}}$ is the corresponding change of $S_{r_{3}}$. It is well known that the perihelion shift originates due to a small relativistic correction $\varepsilon$ to $M^{2}$ in $S_{r_{3}}: \quad M^{2} / r_{3}^{2} \Rightarrow$ $\left(M^{2}-\varepsilon\right) / r_{3}^{2}$. Relation (3.7) shows that, in our case, $\varepsilon=D m^{\prime 2} c^{2} r_{g}^{2} /[2(D-2)]$. Expanding $S_{r_{3}}$ in powers of this correction,

$$
\begin{aligned}
& S_{r_{3}}=S_{r_{3}}\left(M^{2}-\varepsilon\right) \approx S_{r_{3}}^{(0)}-\varepsilon \frac{\partial S_{r_{3}}^{(0)}}{\partial M^{2}}= \\
& =S_{r_{3}}^{(0)}-\frac{\varepsilon}{2 M} \frac{\partial S_{r_{3}}^{(0)}}{\partial M}= \\
& =S_{r_{3}}^{(0)}-\frac{D m^{\prime 2} c^{2} r_{g}^{2}}{4(D-2) M} \frac{\partial S_{r_{3}}^{(0)}}{\partial M}
\end{aligned}
$$

where $S_{r_{3}}^{(0)} \equiv S_{r_{3}}\left(M^{2}\right)$, we obtain

$\Delta S_{r_{3}} \approx \Delta S_{r_{3}}^{(0)}-\frac{D m^{\prime 2} c^{2} r_{g}^{2}}{4(D-2) M} \frac{\partial \Delta S_{r_{3}}^{(0)}}{\partial M}$

Differentiating this equation with respect to $M$, we get

$\Delta \psi \approx 2 \pi+\frac{D \pi m^{\prime 2} c^{2} r_{g}^{2}}{2(D-2) M^{2}}$,

where we took into account that $-\partial \Delta S_{r_{3}}^{(0)} / \partial M=$ $\Delta \psi^{(0)}=2 \pi$. Therefore, the second term in (3.13) gives the required formula for the perihelion shift in our multidimensional case:

$\delta \psi=\frac{D \pi m^{\prime 2} c^{2} r_{g}^{2}}{2(D-2) M^{2}}=\frac{D \pi r_{g}}{(D-2) a\left(1-e^{2}\right)}$.

In this equation, we used the well-known relation $M^{2}=$ $m^{\prime 2} r_{g} c^{2} a\left(1-e^{2}\right) / 2$, with $a$ and $e$ being the semimajor axis and the eccentricity of the ellipse, respectively. In the three-dimensional case $D=3$, this equation exactly coincides with formula (101.7) in [22]. It can be easily seen that result (3.14) does not depend on the motion of the gravitating and test masses in the extra dimensions.

It makes sense to apply this formula to Mercury, because it has the most significant discrepancy in the solar system between the measurement value of perihelion shift and its value calculated with the use of Newton's 
formalism. The observed discrepancy is $43.11 \pm 0.21$ arcsec per century. This missing value is usually explained by the relativistic effects of the form (3.14). However, only in three-dimensional case $D=3$, relation (3.14) gives the satisfactory result $42.94^{\prime \prime}$, which is within the measurement accuracy. For the $D=4$ and $D=9$ models, we obtain $28.63^{\prime \prime}$ and $18.40^{\prime \prime}$, respectively, which are very far from the observable value.

\subsection{Deflection of light}

Let us consider the propagation of light in a gravitational field with metric (2.52). In the case of massless particles, the Hamilton-Jacobi equation (3.3) is reduced to the eikonal equation:

$g^{i k} \frac{\partial \Psi}{\partial x^{i}} \frac{\partial \Psi}{\partial x^{k}}=0$

For metric (2.52), it reads

$\frac{1}{c^{2}}\left(1+\frac{r_{g}}{r_{3}}+\frac{r_{g}^{2}}{2 r_{3}^{2}}+\frac{D v^{2}}{2(D-2) c^{2}} \frac{r_{g}}{r_{3}}\right)\left(\frac{\partial \Psi}{\partial t}\right)^{2}-$

$-\frac{2(D-1) v^{\alpha}}{(D-2) c^{2}} \frac{r_{g}}{r_{3}} \frac{\partial \Psi}{\partial t} \frac{\partial \Psi}{\partial x^{\alpha}}-\left(1-\frac{1}{D-2} \frac{r_{g}}{r_{3}}\right)\left(\frac{\partial \Psi}{\partial r_{3}}\right)^{2}-$

$-\frac{1}{r_{3}^{2}}\left(1-\frac{1}{D-2} \frac{r_{g}}{r_{3}}\right)\left(\frac{\partial \Psi}{\partial \psi}\right)^{2}-\left(1-\frac{1}{D-2} \frac{r_{g}}{r_{3}}\right) \times$

$\times\left[\left(\frac{\partial \Psi}{\partial x^{4}}\right)^{2}+\ldots+\left(\frac{\partial \Psi}{\partial x^{D}}\right)^{2}\right] \approx 0$

where we take into account that light propagates in the orbital plane $\theta=\pi / 2$. The eikonal function $\Psi$ can be written in the form

$$
\begin{aligned}
& \Psi=-\omega_{0} t+\frac{\rho \omega_{0}}{c} \psi+\Psi_{r_{3}}\left(r_{3}\right)+ \\
& +\Psi_{4}\left(x^{4}\right)+\Psi_{5}\left(x^{5}\right)+\ldots+\Psi_{D}\left(x^{D}\right),
\end{aligned}
$$

where $\omega_{0}=-\partial \Psi / \partial t$ is the frequency of light, and $\rho$ is a constant. Later, we will show that $\rho$ is the impact parameter, i.e., the distance of the closest approach of ray's path to the gravitating mass. Taking into account that $k=\omega_{0} / c$ is the absolute value of the wave-vector, it is clear that $M \equiv \rho k=\rho \omega_{0} / c$ plays the role of the angular momentum for the light beam.

We now consider the natural case where light propagates in our three-dimensional space and does not have components of the momentum in the extra dimensions, i.e., $p_{\alpha}=d \Psi_{\alpha} / d x^{\alpha} \equiv 0, \quad \alpha=4, \ldots, D$. Then, from (3.16) and (3.17) up to the order $O\left(1 / c^{4}\right)$, we obtain the following formula:

$$
\begin{aligned}
& \left(\frac{d \Psi_{r_{3}}}{d r_{3}}\right)^{2} \approx \frac{\omega_{0}^{2}}{c^{2}}\left(1-\frac{1}{D-2} \frac{r_{g}}{r_{3}}\right)^{-1} \times \\
& \times\left(1+\frac{r_{g}}{r_{3}}+\frac{r_{g}^{2}}{2 r_{3}^{2}}+\frac{D v^{2}}{2(D-2) c^{2}} \frac{r_{g}}{r_{3}}\right)-\frac{\rho^{2} \omega_{0}^{2}}{c^{2} r_{3}^{2}} \approx \\
& \approx \frac{\omega_{0}^{2}}{c^{2}}\left(1+\frac{D-1}{D-2} \frac{r_{g}}{r_{3}}-\frac{\rho^{2}}{r_{3}^{2}}\right) .
\end{aligned}
$$

Integrating this expression, we get

$$
\Psi_{r_{3}} \approx \frac{\omega_{0}}{c} \int\left(1+\frac{D-1}{D-2} \frac{r_{g}}{r_{3}}-\frac{\rho^{2}}{r_{3}^{2}}\right)^{1 / 2} d r_{3}
$$

Considering the term with $r_{g} / r_{3}$ as a small relativistic correction, we expand the integrand up to the order $O\left(1 / c^{3}\right)$ :

$$
\begin{aligned}
& \Psi_{r_{3}} \approx \Psi_{r_{3}}^{(0)}+\frac{D-1}{2(D-2)} \frac{r_{g} \omega_{0}}{c} \int\left(r_{3}^{2}-\rho^{2}\right)^{-1 / 2} d r_{3}= \\
& =\Psi_{r_{3}}^{(0)}+\frac{D-1}{2(D-2)} \frac{r_{g} \omega_{0}}{c} \operatorname{arccosh} \frac{r_{3}}{\rho}
\end{aligned}
$$

where the non-relativistic (i.e., gravity is absent: $r_{g} \equiv 0$ ) eikonal function is

$$
\begin{aligned}
& \Psi_{r_{3}}^{(0)}=\frac{\omega_{0}}{c} \int\left(1-\frac{\rho^{2}}{r_{3}^{2}}\right)^{1 / 2} d r_{3} \equiv \\
& \equiv \int\left(\left(\frac{\omega_{0}}{c}\right)^{2}-\frac{M^{2}}{r_{3}^{2}}\right)^{1 / 2} d r_{3} .
\end{aligned}
$$

In this non-relativistic approximation, the trajectory of the light beam is a straight line. Indeed, we have in this case (by full analogy with (3.9)):

$$
\frac{\partial \Psi^{(0)}}{\partial M}=\psi^{(0)}+\frac{\partial \Psi_{r_{3}}^{(0)}}{\partial M}=\psi^{(0)}-\arccos \left(\rho / r_{3}\right)=0 .
$$

Here, the constant is taken in such a way that $\psi^{(0)} \rightarrow$ $\pi / 2$ for $r_{3} \rightarrow \infty$. Thus, the trajectory $\rho=r_{3} \cos \psi^{(0)}$ is a straight line. Obviously, in the non-relativistic case, the total change of the angle $\psi^{(0)}$ is $\Delta \psi^{(0)}=$ $-\partial \Delta \Psi_{r_{3}}^{(0)} / \partial M=\pi$.

Coming back to the relativistic case (3.20), the change of the eikonal function for the light beam traveling from some distance $r_{3}=R$ to the closest approach to the gravitating mass at $r_{3}=\rho$ and again to the distance $r_{3}=R$ is

$$
\Delta \Psi_{r_{3}} \approx \Delta \Psi_{r_{3}}^{(0)}+\frac{D-1}{D-2} \frac{r_{g} \omega_{0}}{c} \operatorname{arccosh} \frac{R}{\rho} .
$$


The corresponding change of the polar angle $\psi$ is

$$
\begin{aligned}
& \frac{\partial \Psi}{\partial M}=\psi+\frac{\partial \Psi_{r_{3}}}{\partial M}=\mathrm{const} \Longrightarrow \\
& \Delta \psi=-\frac{\partial \Delta \Psi_{r_{3}}}{\partial M} \approx-\frac{\partial \Delta \Psi_{r_{3}}^{(0)}}{\partial M}+ \\
& +\frac{D-1}{D-2} \frac{r_{g} R}{\rho}\left(R^{2}-\rho^{2}\right)^{-1 / 2} .
\end{aligned}
$$

Thus, in the limit $R \rightarrow+\infty$, we finally get

$\Delta \psi \approx \pi+\frac{D-1}{D-2} \frac{r_{g}}{\rho}$.

Therefore, the second term in (3.25) gives the required formula for the deflection of light in our multidimensional case:

$\delta \psi=\frac{D-1}{D-2} \frac{r_{g}}{\rho}$.

For the three-dimensional case $D=3$, this equation exactly coincides with formula (101.9) in [22].

We now apply this formula to the Sun. Obviously, the radius $R$ of the Sun is much greater than the size of the extra dimensions, and approximation (2.46) works well at the distances $r_{3} \geq R$. For general relativity and for a ray that grazes the Sun's limb, $\delta \psi \approx 1.75 \operatorname{arcsec}$ is in very good agreement with observational data [12]. Relation (3.26) shows that we get this value of $\delta \psi$ only for the usual three-dimensional space. In the cases where $D=4$ and $D=9$, we obtain, respectively, $\delta \psi \approx 1.31^{\prime \prime}$ and $\delta \psi \approx 1.00^{\prime \prime}$, which are very far from the observable value.

\section{Soliton and Black String Metrics}

Our previous analysis demonstrates that the physically reasonable delta-shaped matter source in the multidimensional Kaluza-Klein model results in asymptotic metric coefficients, which do not provide the correct values for the classical gravitational tests if $D>3$. On the other hand, there are a number of well-known exact vacuum solutions for the Kaluza-Klein models. Therefore, it is of interest to determine the relationship between these exact solutions and our asymptotic metric coefficients. In this regard, we will investigate a 5-D static metric in isotropic (with respect to our threedimensional space) coordinates:

$$
\begin{aligned}
& d s^{2}=A\left(r_{3}\right) c^{2} d t^{2}+B\left(r_{3}\right)\left(d x^{2}+d y^{2}+d z^{2}\right)+ \\
& +C\left(r_{3}\right) d \xi^{2}
\end{aligned}
$$

where $r_{3}=\sqrt{x^{2}+y^{2}+z^{2}}$. This spacetime has two Killing vectors $\partial / \partial t$ and $\partial / \partial \xi$. It is clear that the appropriate energy-momentum tensor should not depend on the time $t$ and the fifth coordinate $\xi$. We suppose that metric (4.1) is a solution of the vacuum Einstein equation

$R_{i k}=0$

with the proper boundary conditions.

To our knowledge, the first solution of the form (4.1) in non-isotropic "Schwarzschild-like" coordinates was found in [14] and reads

$$
\begin{aligned}
& d s^{2}=\left(1-\frac{b}{r_{3}^{\prime}}\right)^{a^{\prime}} c^{2} d t^{2}-\left(1-\frac{b}{r_{3}^{\prime}}\right)^{-a^{\prime}-b^{\prime}} d r_{3}^{\prime 2}- \\
& -\left(1-\frac{b}{r_{3}^{\prime}}\right)^{1-a^{\prime}-b^{\prime}} r_{3}^{\prime 2} d \Omega_{2}^{2}-\left(1-\frac{b}{r_{3}^{\prime}}\right)^{b^{\prime}} d \xi^{2}
\end{aligned}
$$

where $a^{\prime}$ and $b^{\prime}$ are constants satisfying the condition

$a^{\prime 2}+a^{\prime} b^{\prime}+b^{2}=1$

and the parameter $b$ is usually connected with the gravitating mass: $a^{\prime} b=2 G_{N} m / c^{2}=r_{g}$. Then, in the isotropic coordinates, this solution was obtained in [15] and [16] and dubbed the soliton solution in the literature. Its generalization for $D \geq 5$ was recently performed in [27]. In our paper, we choose the metric in the parametrization proposed in [16]:

$d s^{2}=\left(\frac{a r_{3}-1}{a r_{3}+1}\right)^{2 \varepsilon k} c^{2} d t^{2}-\left(1-\frac{1}{a^{2} r_{3}^{2}}\right)^{2} \times$

$\times\left(\frac{a r_{3}+1}{a r_{3}-1}\right)^{2 \varepsilon(k-1)}\left(d r_{3}^{2}+r_{3}^{2} d \Omega_{2}^{2}\right)-$

$-\left(\frac{a r_{3}+1}{a r_{3}-1}\right)^{2 \varepsilon} d \xi^{2}$,

where $a, \varepsilon$ and $k$ are constants, and the parameters $\varepsilon$ and $k$ satisfy the condition

$\varepsilon^{2}\left(k^{2}-k+1\right)=1$.

The Schwarzschild-like solution (4.3) and the soliton solution (4.5) are connected by the relations

$r_{3}^{\prime}=r_{3}\left(1+\frac{b}{4 r_{3}}\right)^{2}$

and

$a^{\prime}=\varepsilon k, b^{\prime}=-\varepsilon, a=\frac{4}{b}$

It follows from (4.7) that $r_{3}^{\prime}=r_{3}+O\left(1 / c^{2}\right)$ if $b=4 / a=$ $r_{g} / a^{\prime}$. 
In the approximation $f \equiv 1 /\left(a r_{3}\right) \ll 1$ and up to $O(f)$, we obtain the following formulas for the metric coefficients of (4.5):

$$
\begin{aligned}
& B\left(r_{3}\right)=-\left(1-f^{2}\right)^{2}\left(\frac{1+f}{1-f}\right)^{2 \varepsilon(k-1)} \approx \\
& \approx-1-4 \varepsilon(k-1) f=-1-\frac{4 \varepsilon(k-1)}{a r_{3}}
\end{aligned}
$$

and

$C\left(r_{3}\right)=-\left(\frac{1+f}{1-f}\right)^{2 \varepsilon} \approx-1-4 \varepsilon f=-1-\frac{4 \varepsilon}{a r_{3}}$

Comparing these expressions with our asymptotic metric coefficients from (2.52) (where $D=4$ and $\mathbf{v} \equiv 0$ )

$B\left(r_{3}\right) \approx-1-\frac{r_{g}}{2 r_{3}}, \quad C\left(r_{3}\right) \approx-1-\frac{r_{g}}{2 r_{3}}$,

we get

$k=2, \varepsilon=\frac{1}{\sqrt{3}}, a=\frac{8}{\sqrt{3} r_{g}}$.

Here, we took relation (4.6) into account. Finally, for $A\left(r_{3}\right)$ from (4.5) up to $O\left(f^{2}\right)$, we get

$$
\begin{aligned}
& A\left(r_{3}\right)=\left(\frac{1-f}{1+f}\right)^{2 \varepsilon k} \approx 1-4 \varepsilon k f+8 \varepsilon^{2} k^{2} f^{2}= \\
& =1-\frac{4 \varepsilon k}{a r}+\frac{8 \varepsilon^{2} k^{2}}{a^{2} r^{2}}=1-\frac{r_{g}}{r}+\frac{r_{g}^{2}}{2 r^{2}}
\end{aligned}
$$

in complete analogy with the asymptotic metric coefficient $A\left(r_{3}\right)$ in (2.52). Therefore, for parameters (4.12), the soliton metric (4.5) reads

$$
\begin{aligned}
& d s^{2}=\left(\frac{1-\sqrt{3} r_{g} / 8 r_{3}}{1+\sqrt{3} r_{g} / 8 r_{3}}\right)^{\frac{4}{\sqrt{3}}} c^{2} d t^{2}- \\
& -\left(1-3 r_{g}^{2} / 64 r_{3}^{2}\right)^{2}\left(\frac{1+\sqrt{3} r_{g} / 8 r_{3}}{1-\sqrt{3} r_{g} / 8 r_{3}}\right)^{\frac{2}{\sqrt{3}}} \times \\
& \times\left(d r_{3}^{2}+r_{3}^{2} d \Omega_{2}^{2}\right)-\left(\frac{1+\sqrt{3} r_{g} / 8 r_{3}}{1-\sqrt{3} r_{g} / 8 r_{3}}\right)^{\frac{2}{\sqrt{3}}} d \xi^{2} .
\end{aligned}
$$

Our analysis shows that this form of the metric provides the correct asymptotic behavior in the case of a deltashaped matter source. Metric (4.14) is the exact solution of the Einstein equation for the gravitating mass at rest $(\mathbf{v} \equiv 0)$ "smeared" uniformly over the extra dimension. The only non-zero component of the energy-momentum tensor is $T_{00}$. We can prove it in the following way. It is clear from the previous consideration that the metric coefficients in (4.14) up to the terms $1 / c^{2} \mathrm{read}$

$$
\begin{aligned}
& g_{00} \approx 1-\frac{r_{g}}{r_{3}}=1+h_{00}, \\
& g_{\alpha \alpha} \approx-1-\frac{r_{g}}{2 r_{3}}=-1+h_{\alpha \alpha} \Rightarrow \\
& h_{00}=-\frac{r_{g}}{r_{3}}, \quad h_{\alpha \alpha}=-\frac{r_{g}}{2 r_{3}}, \quad \alpha=1,2,3,4 .
\end{aligned}
$$

With the same accuracy, the components of the Ricci tensor are

$$
\begin{aligned}
& R_{00} \approx \frac{1}{2} \triangle h_{00}=-\frac{1}{2} \triangle \frac{r_{g}}{r_{3}}=-\frac{G_{N} m}{c^{2}} \triangle \frac{1}{r_{3}}= \\
& =\frac{4 \pi G_{N} m \delta\left(\mathbf{r}_{3}\right)}{c^{2}}=k_{N} \frac{1}{2} m \delta\left(\mathbf{r}_{3}\right) c^{2}, \\
& R_{\alpha \alpha} \approx k_{N} \frac{1}{4} m \delta\left(\mathbf{r}_{3}\right) c^{2}, \quad \alpha=1,2,3,4,
\end{aligned}
$$

where $k_{N} \equiv 8 \pi G_{N} / c^{4}$, and the operator $\triangle$ is defined in (2.13). Taking into account that the matter source is at rest, we may conclude from (4.16) that the only non-zero component of the energy momentum tensor is $T_{00}$. Hence, for the Einstein equations, we obtain

$$
\begin{aligned}
& R_{00}=k^{\prime \prime}\left(T_{00}-\frac{1}{3} T g_{00}\right)=k^{\prime \prime} \frac{2}{3} T_{00}, \\
& R_{\alpha \alpha}=k^{\prime \prime}\left(T_{\alpha \alpha}-\frac{1}{3} T g_{\alpha \alpha}\right) \approx \\
& \approx k^{\prime \prime} \frac{1}{3} T_{00}, \quad \alpha=1,2,3,4,
\end{aligned}
$$

where $k^{\prime \prime} \equiv 2 S_{D} \tilde{G}_{\mathcal{D}} / c^{4}$ in accordance with (2.3). Therefore, from (4.16) and (4.17) up to the terms $c^{2}$, we get

$T_{00} \approx \frac{k_{N}}{k^{\prime \prime}} \frac{3}{4} m \delta\left(\mathbf{r}_{3}\right) c^{2}=\frac{1}{a_{1}} m \delta\left(\mathbf{r}_{3}\right) c^{2}$,

where $a_{1}$ is the size of the extra dimension (the period of the torus), and we take the relations $G_{\mathcal{D}}=$ $[2(D-2) /(D-1)] \tilde{G}_{\mathcal{D}}$ and $S_{D} G_{\mathcal{D}} / \prod_{\alpha=1}^{d} a_{\alpha}=4 \pi G_{N}$ into account. We can write (4.18) in the form $T_{00} \approx \rho c^{2}$, where $\rho=\left(m / a_{1}\right) \delta\left(\mathbf{r}_{3}\right)$ in accordance with (2.47). This energy momentum tensor has a delta-shaped form. However, we can generalize this consideration to the case of a finite-size spherically symmetric gravitating body at rest smeared uniformly over the fifth compact dimension. Analyzing the Einstein equations (where we should keep the terms up to $1 / c^{2}$ ), we can conclude that the metric coefficients outside of the body should take the form (4.15). In this case, $T_{00} \equiv 0$ and $T_{00} \approx m c^{2} /\left(V_{3} a_{1}\right)$ outside and inside the body, respectively, and $V_{3}$ is the three-dimensional part of the body volume. 
All others soliton metrics (4.5) with the parameters different from (4.12) have no correspondence with the asymptotic metric (2.52). Besides the non-zero component $T_{00}$, these models will also have some other nonzero components of the energy-momentum tensor, which have no clear physical origin. It is worth noting that, in works $[5,6]$, the classical tests of general relativity were applied to the soliton solutions, and it was shown that these models do not contradict the observations if the modulus of the parameter $\varepsilon$ in (4.5) is at most 0.07 . Obviously, this value is much less than $\varepsilon=1 / \sqrt{3} \approx 0.58$ in (4.12). This result confirms the conclusion of the previous section that the multidimensional Kaluza-Klein models with a physical reasonable source of matter (with smeared or not smeared extra dimensions) do not satisfy the experimental data.

There is one interesting particular example satisfying the condition $|\varepsilon|<0.07$ which corresponds in (4.5) to the limit

$\varepsilon \rightarrow 0, k \rightarrow+\infty, \varepsilon k \rightarrow 1$,

or to the limit $a^{\prime} \rightarrow 1, b^{\prime} \rightarrow 0$ in the Schwarzschild-like metric (4.3). In this limit, metric (4.5) reads

$$
\begin{aligned}
& d s^{2}=\left(\frac{a r_{3}-1}{a r_{3}+1}\right)^{2} c^{2} d t^{2}-\left(\frac{a r_{3}+1}{a r_{3}}\right)^{4} \times \\
& \times\left(d r_{3}^{2}+r_{3}^{2} d \Omega_{2}^{2}\right)-d \xi^{2} .
\end{aligned}
$$

It can be easily seen that the four-dimensional part of this metric (which corresponds to the section $\xi=$ const) is the pure Schwarzschild metric (for $a=4 / r_{g}$ ) in isotropic coordinates. Metrics of the form (4.20) are often called the uniform black strings. From this metric up to the terms $1 / c^{2}$, we get

$$
\begin{aligned}
& g_{00} \approx 1-\frac{r_{g}}{r_{3}}=1+h_{00}, \\
& g_{\alpha \alpha} \approx-1-\frac{r_{g}}{r_{3}}=-1+h_{\alpha \alpha}, \quad \alpha=1,2,3, \\
& g_{44}=-1=-1+h_{44} \Rightarrow \\
& h_{00}=h_{11}=h_{22}=h_{33}=-\frac{r_{g}}{r_{3}}, \quad h_{44}=0 .
\end{aligned}
$$

With the same accuracy, the components of the Ricci tensor are

$$
\begin{aligned}
& R_{00} \approx \frac{1}{2} \triangle h_{00}=-\frac{1}{2} \triangle \frac{r_{g}}{r_{3}}=-\frac{G_{N} m}{c^{2}} \triangle \frac{1}{r_{3}}= \\
& =\frac{4 \pi G_{N} m \delta\left(\mathbf{r}_{3}\right)}{c^{2}}=k_{N} \frac{1}{2} m \delta\left(\mathbf{r}_{3}\right) c^{2}, \\
& R_{11}=R_{22}=R_{33} \approx k_{N} \frac{1}{2} m \delta\left(\mathbf{r}_{3}\right) c^{2}, \\
& R_{44}=0 .
\end{aligned}
$$

For a gravitating mass at rest in view of (4.22), we arrive at the conclusion that $T_{00}$ and $T_{44}$ are the only non-zero components of the energy momentum tensor. Thus, the Einstein equations take the form

$$
\begin{aligned}
& R_{00}=k^{\prime \prime}\left(T_{00}-\frac{1}{3} T g_{00}\right) \\
& R_{11}=R_{22}=R_{33}=k^{\prime \prime}\left(-\frac{1}{3} T g_{11}\right) \\
& R_{44}=k^{\prime \prime}\left(T_{44}-\frac{1}{3} T g_{44}\right)
\end{aligned}
$$

Therefore, from (4.22) and (4.23) up to the terms $c^{2}$, we get

$$
\begin{aligned}
& T_{00} \approx \frac{k_{N}}{k^{\prime \prime}} m \delta\left(\mathbf{r}_{3}\right) c^{2}=\frac{4}{3} \frac{m}{a_{1}} \delta\left(\mathbf{r}_{3}\right) c^{2} \\
& T_{44} \approx-\frac{1}{2} \frac{k_{N}}{k^{\prime \prime}} m \delta\left(\mathbf{r}_{3}\right) c^{2}=-\frac{2}{3} \frac{m}{a_{1}} \delta\left(\mathbf{r}_{3}\right) c^{2} \\
& T=T_{00} g^{00}+T_{44} g^{44} \approx \\
& \approx \frac{3}{2} \frac{k_{N}}{k^{\prime \prime}} m \delta\left(\mathbf{r}_{3}\right) c^{2}=2 \frac{m}{a_{1}} \delta\left(\mathbf{r}_{3}\right) c^{2}
\end{aligned}
$$

The presence of the non-zero component $T_{44}$ results in a non-vanishing tension of black strings (see, e.g., $[18,19])$. Relation (4.24) shows that the value $T_{00}$ is two times bigger than the modulus of $T_{44}$. A similar relation exists for the ADM mass and the tension (see, e.g., (B.11) and (B.12) in [18]). For the soliton solution (4.14), the tension is absent. This follows from the fact that $T_{44} \equiv 0$, or we can see it also from (B.12) in [18], where we should insert the correction terms $h_{i k}$ from our relation (4.15).

Thus, we can conclude the following. The black string solutions (4.20) do not contradict the classical gravitational tests. However, the matter sources for these metrics have non-zero tension. It is hard to imagine that astrophysical objects, e.g. the Sun, can have such energy momentum tensor. On the other hand, it is natural to suppose that, for gravitating masses (e.g., ordinary astrophysical objects) at rest, $T_{00}$ is the only non-zero component of the energy momentum tensor. As we have seen above, the soliton solution (4.14) has the matter source with such clear physical interpretation. Moreover, the metric coefficients for this solution have the asymptotic form, which corresponds to the correct non-relativistic Newtonian limit for a gravitating mass. However, analysis conducted in the previous section shows that this multidimensional Kaluza-Klein metric contradicts the experimental data. 


\section{Conclusion and Discussion}

In our paper, we have investigated the classical gravitational tests (frequency shift, perihelion shift, and deflection of light) for multidimensional models with compact internal spaces in the form of torus. We have supposed that, in the absence of gravitating masses, the metric is a flat one. Gravitating masses (moving or at rest) perturb this metric, and we have considered these perturbations in a weak-field approximation. In this approximation, we obtained the asymptotic form of the metric coefficients. Up to this point, we did not require the compactness of extra dimensions. This approach is valid for any number of spatial dimensions $D \geq 3$ and generalizes the well-known calculations [22] in the fourdimensional spacetime. Then, we have admitted that, first, the extra dimensions are compact and have the topology of a torus and, second, the gravitational potential far away from gravitating masses tends to the non-relativistic Newtonian limit. It gave us a possibility to specify the non-relativistic gravitational potential for the considered models. In turn, this enabled us to specify the metric coefficients. In the case of a gravitating body at rest, we used these metric coefficients to calculate the frequency shift, perihelion shift, and deflection of light. We have demonstrated that, for the frequency shift type experiment, it is hardly possible to observe the difference between the usual four-dimensional general relativity and the multidimensional Kaluza-Klein models. However, the situation is quite different for the perihelion shift and the deflection of light. In these two cases, we obtained formulas, which generalize the corresponding ones in general relativity. We have shown that both of these formulas (for perihelion shift and deflection of light) depend on a total number of spatial dimensions $D$, and they are in good agreement with observations only in the ordinary three-dimensional space $D=3$. This result does not depend explicitly on the size of the extra dimensions. Therefore, it is impossible to avoid the problem with classical gravitational tests in a limit of arbitrary small sizes of the extra dimensions.

As was mentioned in Introduction and Section 4, there is a range of parameters for the soliton solutions (4.5) (or, equivalently, (4.3)), for which the classical gravitational tests satisfy the observational values. However, this range of parameters is quite far from the values, which enable the metric coefficients to have the asymptotic form (2.52) corresponding to the correct non-relativistic Newtonian limit for a gravitating mass. Moreover, in this case, the energy momentum tensor has the form that is hard to imagine for ordinary as- trophysical objects. For example, the black string solutions belong to this class of the parameters and have matter sources with non-zero tension. It is very difficult to explain how the astrophysical objects, e.g. the Sun, can have such energy momentum tensor. On the other hand, we have found the exact soliton solution (4.14) with metric coefficients of the asymptotic form (2.52). Here, the matter source has a delta-shaped form, and $T_{00}$ is the only non-zero component of the energy momentum tensor. Such energy momentum tensor has a clear physical interpretation for a gravitating mass at rest. However, our analysis demonstrates that this multidimensional Kaluza-Klein metric contradicts the experimental data. So, we arrive at the following situation: either we have the metrics (e.g., the black string (4.20)), which do not contradict the observations, but have to deal with very unnatural (for ordinary astrophysical objects) energy momentum tensors for matter sources, or we have the metrics (e.g., our soliton solution (4.14)), which correspond to physically reasonable matter sources, but contradict the experimental data.

Therefore, the considered multidimensional KaluzaKlein models face a severe problem. This testifies to the need for a strong reconsideration of the foundations of extra-dimensional models. A possible resolution might be provided in models with branes or with a non-linear action $f(R)$. However, to prove the viability of these models, it is necessary to perform the similar investigation.

We want to thank Uwe Günther for useful comments. This work was supported in part by the "Cosmomicrophysics" program of the Division of Physics and Astronomy of the National Academy of Sciences of Ukraine.

1. Th. Kaluza, Sitzungsber. d. Preuss. Akad. d. Wiss., 966 (1921); O. Klein, Zeit. für Physik 37, 895 (1926).

2. J. Polchinski, String Theory, Volume 2: Superstring Theory and Beyond (Cambridge Univ. Press, Cambridge, 1998).

3. G. Bhattacharyya, A. Datta, S.K. Majee, and A. Raychaudhuri, Nucl. Phys. B 821, 48 (2009); arXiv:hep$\mathrm{ph} / 0904.0937$.

4. D. Kalligas, P.S. Wesson, and C.W.F. Everitt, Astrophys. J. 439, 548 (1995).

5. P.H. Lim, J.M. Overduin, and P.S. Wesson, J. Math. Phys. 36, 6907 (1995).

6. H. Liu and J. Overduin, Astrophys. J. 538, 386 (2000); arXiv:gr-qc/0003034.

7. T. Liko, J.M. Overduin, and P.S. Wesson, Space Sci. Rev. 110, 337 (2004); arXiv:gr-qc/0311054. 
8. F. Rahaman, S. Ray, M. Kalam, and M. Sarker, Int. J. Theor. Phys. 48, 3124 (2009); arXiv:gr-qc/0707.0951.

9. P. Xu and Y. Ma, Phys. Lett. B 656, 165 (2007); arXiv:gr-qc/0710.3677.

10. N.J. Poplawski, Einstein-Cartan gravity excludes extra dimensions (2010); arXiv:hep-th/1001.4324.

11. I.I. Shapiro, W.B. Smith, M.E. Ash, and S. Herrick, Astron. J. 76, 588 (1971); I.I. Shapiro, C.C. Counselman, and R.W. King, Phys. Rev. Lett. 36, 555 (1976).

12. S.S. Shapiro, J.L. Davis, D.E. Lebach, and J.S. Gregory, Phys. Rev. Lett. 92, 121101 (2004).

13. R.C. Myers and M.J. Perry, Ann. Phys. 172, 304 (1986).

14. D. Kramer, Acta Phys. Polon. B 2, 807 (1970).

15. D.J. Gross and M.J. Perry, Nucl. Phys. B 226, 29 (1983).

16. A. Davidson and D. Owen, Phys. Lett. 155, 247 (1985).

17. R. Gregory and R. Laflamme, Phys. Rev. Lett. 70, 2837 (1993); arXiv:hep-th/9301052.

18. T. Harmark and N.A. Obers, JHEP 0405, 043 (2004); arXiv:hep-th/0403103.

19. D. Kastor and J. Traschen, JHEP 0609, 022 (2006); arXiv:hep-th/0607051.

20. C.G. Boehmer, T. Harko and F.S.N. Lobo, Class. Quant. Grav. 25, 045015 (2008); arXiv:gr-qc/0801.1375.

21. A. Chodos and S. Detweiler, Gen. Rel. Grav. 14, 879 (1982).

22. L.D. Landau and E.M. Lifshitz, The Classical Theory of Fields (Pergamon Press, Oxford, 2000), Vol. 2.

23. M. Eingorn and A. Zhuk, Phys. Rev. D 80, 124037 (2009); arXiv:hep-th/0907.5371.

24. M. Eingorn and A. Zhuk, Class. Quant. Grav. 27, 055002 (2010); arXiv:gr-qc/0910.3507.

25. D.J. Kapner, T.S. Cook, E.G. Adelberger, J.H. Gundlach, B.R. Heckel, C.D. Hoyle, and H.E. Swanson, Phys. Rev. Lett. 98, 021101 (2007); arXiv:hep-ph/0611184.
26. L.D. Landau and E.M. Lifshitz, Mechanics (Pergamon Press, Oxford, 2000).

27. J.P. de Leon, Grav. Cosmol. 15, 345 (2009); arXiv:grqc/0905.2010; J.P. de Leon, Schwarzschild-like exteriors for stars in Kaluza-Klein gravity (2010); arXiv:grqc/1003.3151.

Received 15.07.11

\section{КЛАСИЧНІ ТЕСТИ БАГАТОВИМІРНОЇ ГРАВІТАЦІЇ НЕГАТИВНИЙ РЕЗУЛЬТАТ}

M. Ейнгорн, А. Жук

$\mathrm{P}$ е $з$ ю м е

У моделі Калуци-Клейна з тороїдальними додатковими вимірами ми отримуємо метричні коефіцієнти для дельтаподібних матеріальних джерел у наближенні слабкого поля. Ці метричні коефіцієнти застосовані для знаходження формул для частотного зсуву, зсуву перигелію та відхилення світла. У головному порядку наближення формула для частотного зсуву збігається з широко відомим виразом загальної теорії відносності. Однак для зсуву перигелію та відхилення світла отримуємо формули $D \pi r_{g} /\left[(D-2) a\left(1-e^{2}\right)\right]$ та $(D-1) r_{g} /[(D-2) \rho]$ відповідно, де $D$ - загальна кількість просторових вимірів. Ці вирази демонструють добре узгодження з експериментальними даними лише у випадку звичайного тривимірного $(D=3)$ простору. Цей результат не залежить від розміру додаткових вимірів. Ми також отримуємо точний 5-D солітонний розв'язок $з$ правильною нерелятивістською ньютонівською границею. Тензор енергії-імпульсу для цього розв'язку має чітку фізичну інтерпретацію. Тим не менш, класичні тести для цієї метрики не задовільняють експериментальні дані. Отже, розглянуті багатовимірні моделі Калуци-Клейна стоять перед серйозною проблемою. 